NASA Contractor Report 3946

\title{
Precision Pointing Using a Dual-Wedge Scanner
}

Christopher T. Amirault and Charles A. DiMarzio

CONTRACT NAS8-33120

NOVEMBER 1985

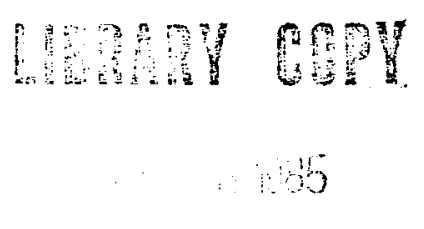

LAWUG RESERCH CERTER

- tobrovisa

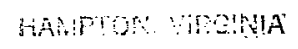




\section{NASA Contractor Report 3946}

\section{Precision Pointing Using a Dual-Wedge Scanner}

Christopher T. Amirault and Charles A. DiMarzio

Raytbeon Company

Sudbury, Massacbusetts

Prepared for

George C. Marshall Space Flight Center under Contract NAS8-33120

\section{NASA


This Page Intentionally Left Blank 


\section{LIST OF ILLUSTRATIONS}

Figure

Page

1 The iterative calibration routine uses trial and error to compare the set of 4 measured data points with trial data points calculated with aircraft orientation, scanner setting, and scanner alignment data.

2 In the dual-wedge scanner coordinate system, the input vector $S$ is collinear with the $x$ axis, which is the axis of rotation for the two wedges.

$3 \quad$ Using simple approximation, counterrotating two equal wedges of the scanner produces a straight line on the $z$ axis.

4 A closer look at counterrotating equal wedges shows errors of 30 meters at 10,000 meters for a scan 8,000 meters wide.

$5 \quad$ Counterrotating unequal wedges produces greater distortions to the scan generated.

6 Rotating the second wedge independently produces the ellipse shown. The desired point can then be arrived at by rotating the wedges simultaneous iy.

7 Calculating aircraft alignment involves three rotations about the coordinate systems of heading, pitch and roll.

8 As the iterations progress, each of the parameters approaches its correct value.

9 Results better than 14 and 10 meters at 10,000 meters are acquired with the two misaligned parameter sets if the rotation angles are unrounded.

10 Due to the one-tenth degree quantization in positioning the scanner, the actual errors are 15 and 12 meters at 10,000 meters. 


\section{LIST OF ILLUSTRATIONS (Cont'd)}

\section{Figure}

Page

11 Fore and aft looking scans create a grid of 28 wind velocity returns in the Severe Storms measurement system.

12 The acquisition and selection of data points is restricted by scanner coverage, physical obstructions, and human limitations. 


\section{INTROOUCTION}

The ability to precisely position a laser beam along one of many desired lines of sight is vital to many laser radar systems. It is often beneficial to house the equipment in a small package as we11, so that, for example, the dimensions of an output window which the scanned beam passes through may be made as small as possible. When limited spatial coverage is sufficient and this packaging constraint exists, a scanner using two wedges which may be rotated independently about the direction of the input beam is an attractive choice. A 30.5-cm-diameter germanium dual-wedge scanner has been built for a $\mathrm{CO}_{2}$ doppler lidar used on NASA's CV-990 aircraft for meteorological research ${ }^{2}$ and was tested on that aircraft during a flight test in $1981 .^{2}$ The success of this test demonstrated that precise, reproducible pointing could be achieved with this device. Two problems were observed during this test and the subsequent analysis:

(a) The dependence of the deviation on the angle of incidence at the second wedge, while small, was sufficient to produce an error greater than that caused by other sources, and more accurate positioning calculations were therefore required.

(b) A more accurate alignment procedure was required to establish the relationship between the scanner axes and a known reference frame. Since the desired reference frame was determined by the inertial 
navigation system (INS) of the aircraft and no reference surfaces were available near the scanner, it became obvious that this alignment must be performed relative to the INS-indicated aircraft orientation.

If the scanner alignment parameters-wedge angles, wedge orientations, and the axes of the scanner and input beam directionare known exactly, the line of sight of an output beam can be determined from the indicated wedge positions as shown in Figure 1(a). This calculation makes use of Snell's law in vector form ${ }^{3}$ and coordinate rotation matrices. More frequently, it is desired to position the wedges to produce a specified line of sight as shown in Figure 1(b). This can be accomplished with only slightly greater difficulty as will be shown later.

Before either of these calculations can be performed, it is necessary to determine the alignment of the scanner. In principle, if enough combinations of measured lines of sight and indicated wedge positions are determined, a set of simultaneous equations can be generated and solved for all the unknowns among the alignment data as shown in Figure $1(c)$. This is accomplished by locating the beam on retroreflector targets at surveyed points and recording the indicated wedge positions which produced each known line of sight. In practice, the inversion process is difficult, and is solved by an iterative approach as shown in Figure 1(d). 


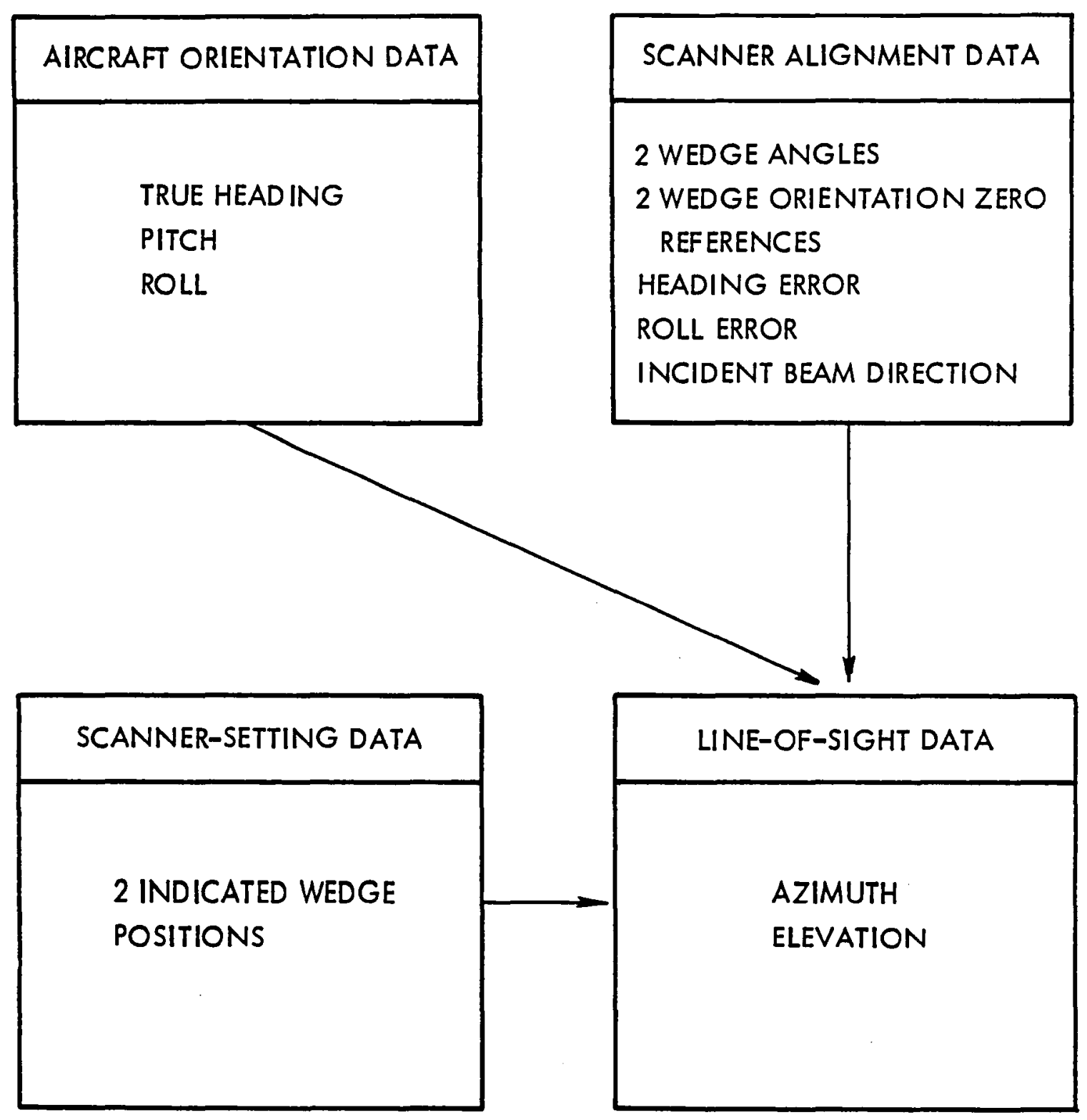

(a) Calculating the Line of Sight

Figure 1. The iterative calibration routine uses trial and error to compare the set of 4 measured data points with trial data points calculated with aircraft orientation, scanner setting, and scanner alignment data. 


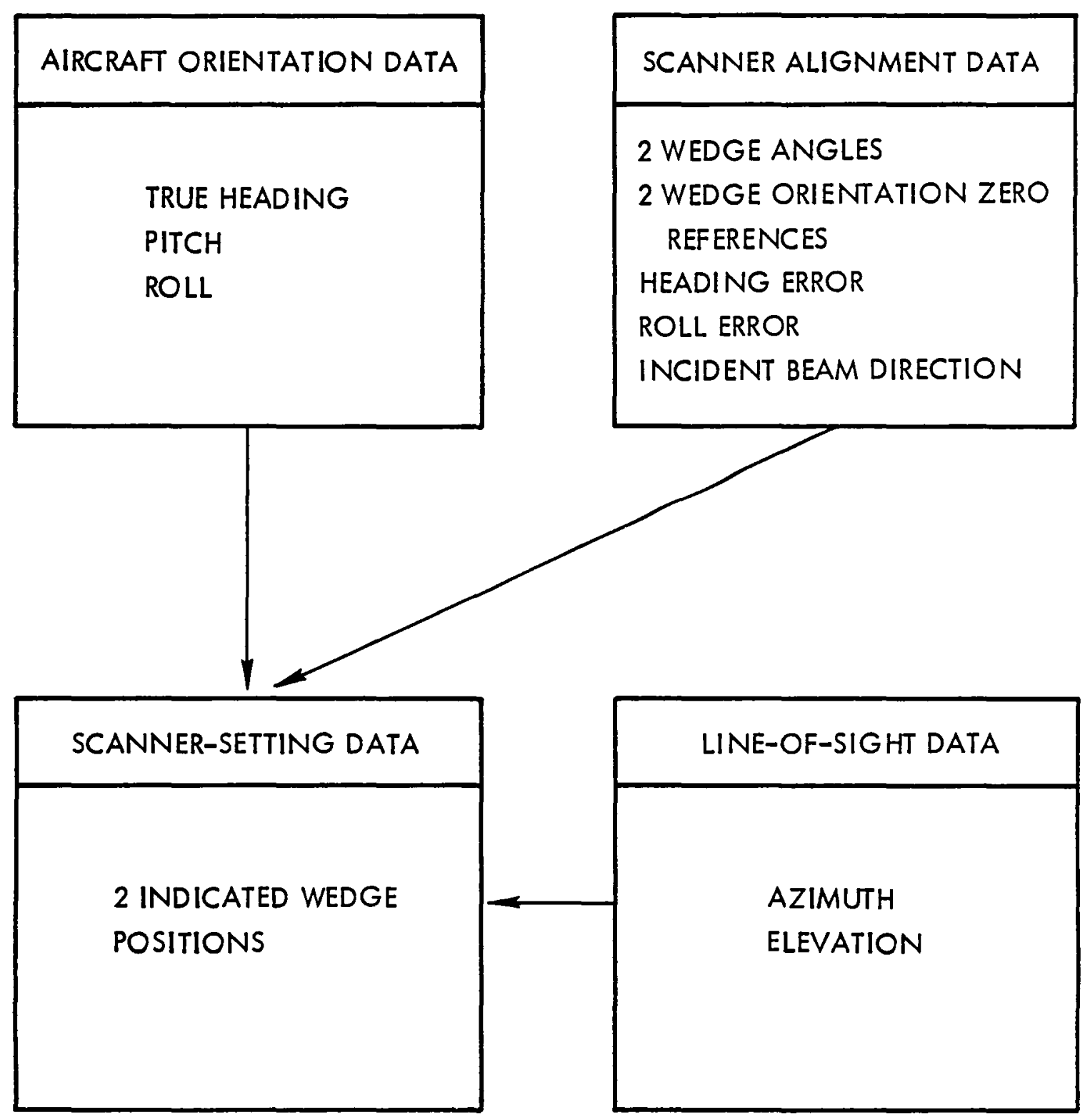

(b) Setting the Scanner to a Line of Sight

Figure 1. The iterative calibration routine uses trial and error to compare the set of 4 measured data points with trial data points calculated with aircraft orientation, scanner setting, and scanner alignment data. 


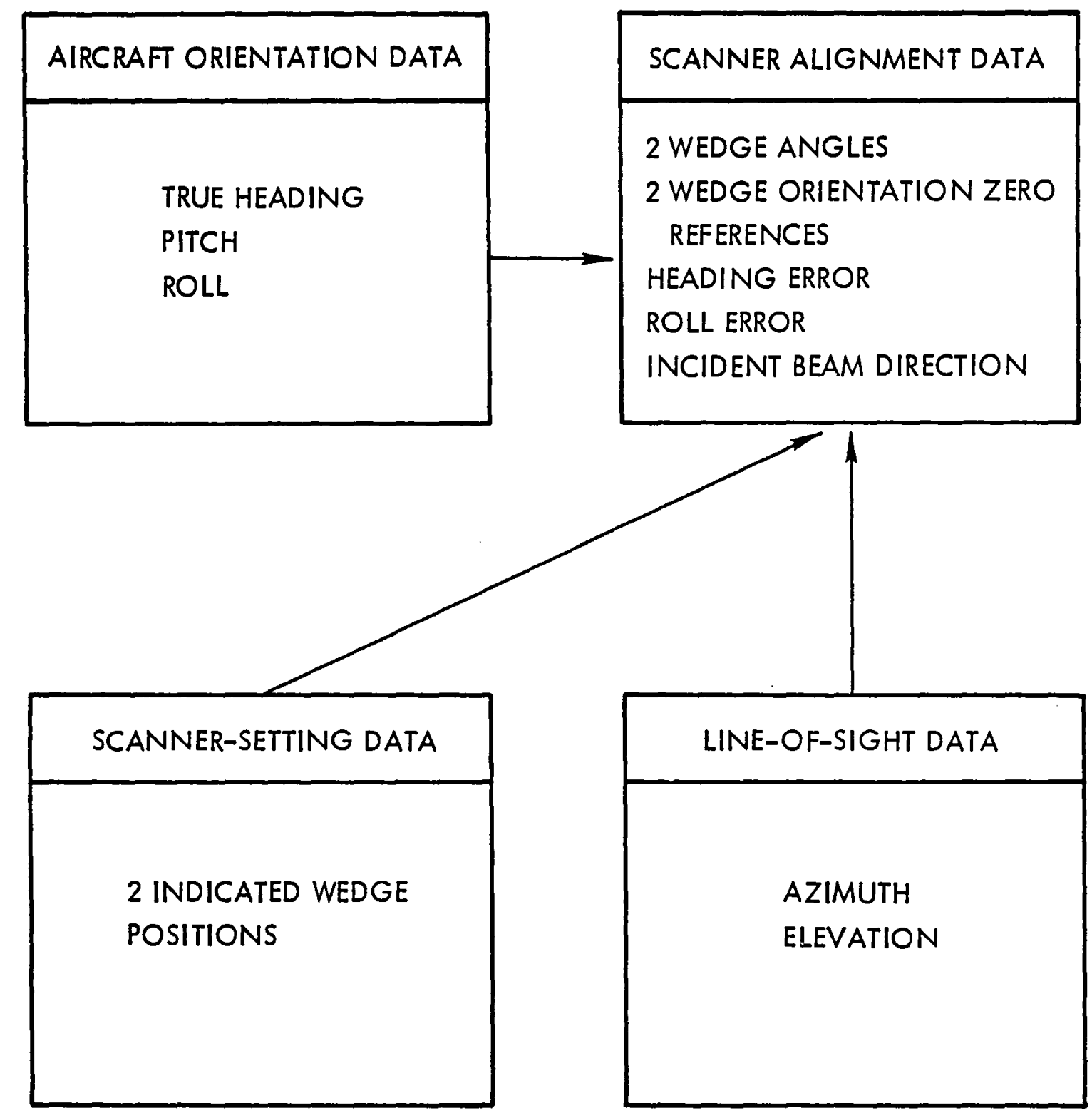

(c) Calibrating the Scanner

Figure 1. The iterative calibration routine uses trial and error to compare the set of 4 measured data points with trial data points calculated with aircraft orientation, scanner setting, and scanner alignment data. 


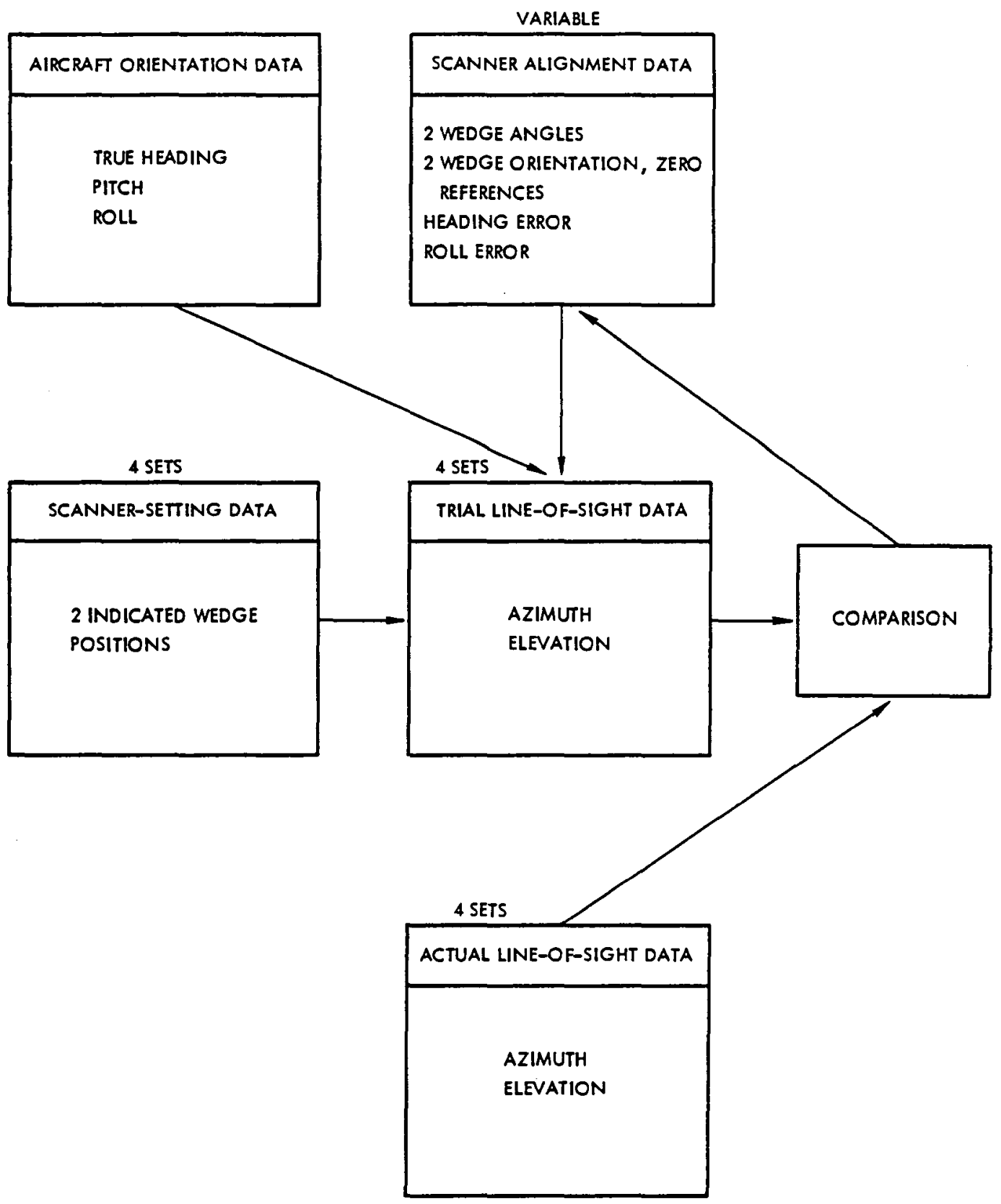

(d) Iterative Calibration Routine

Figure 1. The iterative calibration routine uses trial and error to compare the set of 4 measured data points with trial data points calculated with aircraft orientation, scanner setting, and scanner alignment data. 
The approach to precision pointing will be described beginning with the generation of a simple line scan, followed by a description of the full-coverage pointing algorithm. Next, the iterative calibration algorithm will be described along with its application to a model scanner. Finally, the calibration algorithm will be applied to a limited set of calibration data collected during preparation for the $1981 \mathrm{flight}$ tests, and the results compared to actual flight test results.

\section{LINE SCANS}

One simple application of a dual-wedge scanner is the generation of straight 1 ine scans. As shown in Figure 2, this scanner uses two wedges which have wedge angles that are as nearly equal as possible arranged with their flat sides together. The first wedge produces a certain deflection of the beam in the plane of incidence. As the wedge rotates about the beam direction, the plane of incidence, and thus the plane in which the output beam emerges, rotates as well. If the $x$ axis is defined as collinear to the beam and is thus the axis of rotation as the first wedge is rotated through $360^{\circ}$, a point at a specified range along the deflected beam traverses a circle parallel to the $y-z$ plane centered on the $x$ axis (Figure 3 , box 1 ). With this wedge at $90^{\circ}$, the second wedge, $W_{2}$, is introduced. If the deviation were independent on the angle of incidence, a rotation of $W_{2}$ through $360^{\circ}$ would produce a second circle centered on the first circle 


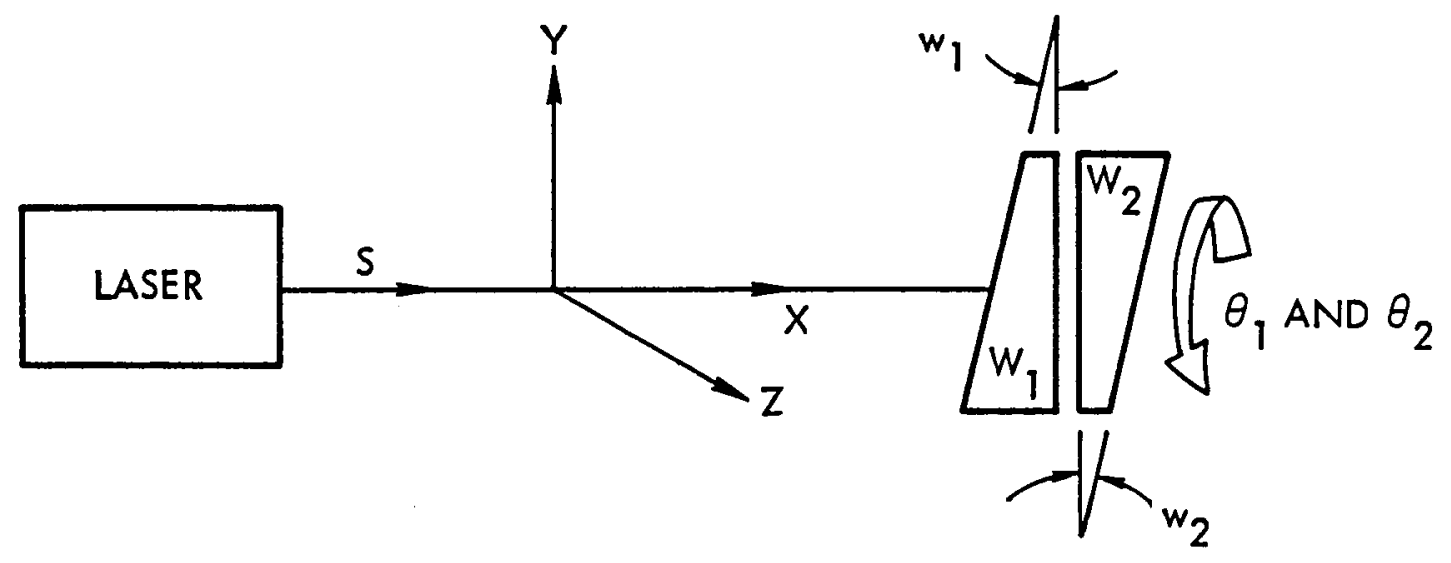

Figure 2. In the dual-wedge scanner coordinate system, the input vector $S$ is collinear with the $x$ axis, which is the axis of rotation for the two wedges. 


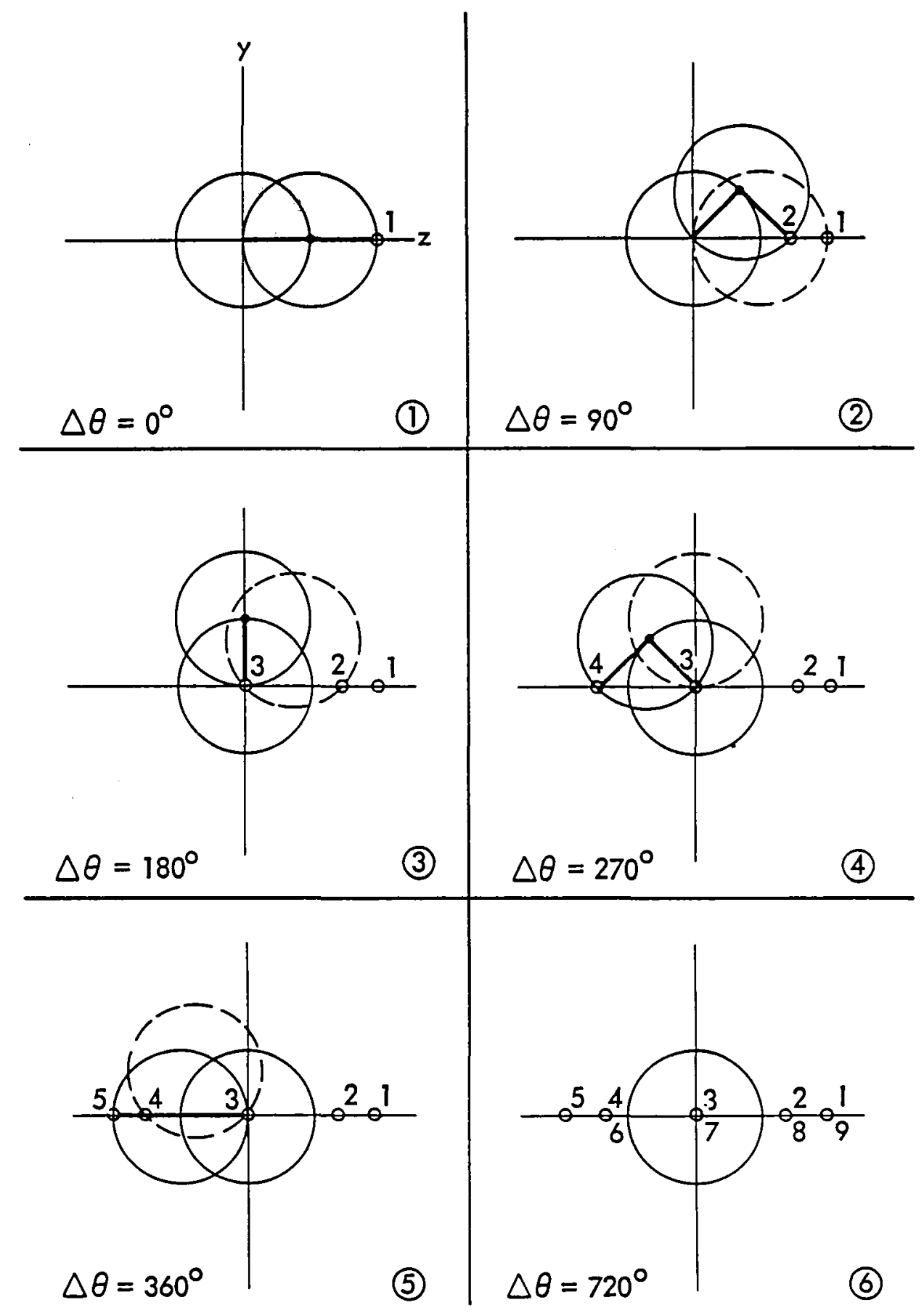

Figure 3. Using simple approximation, counterrotating two equal wedges of the scanner produces a straight line on the $z$ axis. 
at $\theta_{1}=90^{\circ}$. For moderate wedge angles, this is approximately true, and was, in fact, assumed during the 1981 tests. With $\theta_{1}=90^{\circ}$ and $\theta_{2}=90^{\circ}$, the first point of the scan would be at maximum $z$ or twice the radius of the circles out from the origin. Next, the wedges would be counterrotated $45^{\circ}$ each, $\theta_{1}$ counterclockwise and $\theta_{2}$ clockwise looking along the direction of the impact beam until $\Delta \theta=90^{\circ}$ (box 2), and the beam would be at point 2 on the $z$ axis. At $\Delta \theta=180^{\circ}$, point 3 , which coincides with the origin, is reached, and further counterrotation moves the beam to the minimum $z$ at point 5 . Another $360^{\circ}$ results in complete counterrotation and a return to the maximum $z$ value. In this way, a straight line scan of length equal to twice the diameter of the circles would be produced.

However, while the angle of incidence at $W_{1}$ is constant for all rotation angles, it is not at $W_{2}$ since the beam is refracted by $W_{1}$ in one of its many possible orientations. For this reason, the beam path in this scan is not precisely straight. The exact form can be obtained using the vector form of Snell's law, 3

$$
\left.\hat{S}_{j+1}=r_{j} \hat{S}_{j}+\left\{\sqrt{1-r_{j}^{2}\left[1-\left(S_{j} \cdot N_{j}\right)^{2}\right.}\right]-r_{j} \hat{S}_{j} \cdot \hat{N}_{j}\right\} \hat{N}_{j},
$$

where at surface $j$, the vector $S_{j}$ is a unit vector parallel to the incident beam, $S_{j+1}$ is parallel to the refracted beam, $N$ is normal to the surface, and $r_{j}=N_{j} / N_{j+1}$ is the ratio of the index of refraction on the side with the incident beam to that on the side with the refracted beam. If the inner wedge surfaces are 
parallel, they may be neglected; therefore, the two surfaces to be considered are the inner surfaces of the inner wedge and the outer surface of the outer wedge, described by

$$
\hat{N}_{j}=\cos w_{j} \hat{i}+\sin w_{j}\left(\cos \theta_{j} \hat{j}+\sin \theta_{j} \hat{k}\right) \text {, }
$$

with a beam incident along the $x$ axis described by

$$
\hat{s}_{1}=\hat{i} \text {. }
$$

For equal wedges, the scan pattern follows the bow-tie curve shown in Figure 4. This curve, drawn with $w_{1}=w_{2}=3.3275^{\circ}$, shows that the errors in the $y$ direction can equal approximately 30 meters at a range of 10,000 meters. This error reduces to zero whenever $\theta_{1}$ and $\theta_{2}$ are each at $90^{\circ}$ intervals. Figure 5 shows the effects of unequal wedges on the curves and illustrates some of the problems associated with precise positioning using a dual-wedge scanner.

\section{FULL COVERAGE PRECISE POINTING}

The problem of precise pointing could be solved by inverting the vector equations for refraction at two surfaces to solve for $\theta_{1}$ and $\theta_{2}$, but this is a difficult or impossible task. A realistic approach has been developed using a two-step process which may be described in terms of its deviation angle from the $x$ axis 


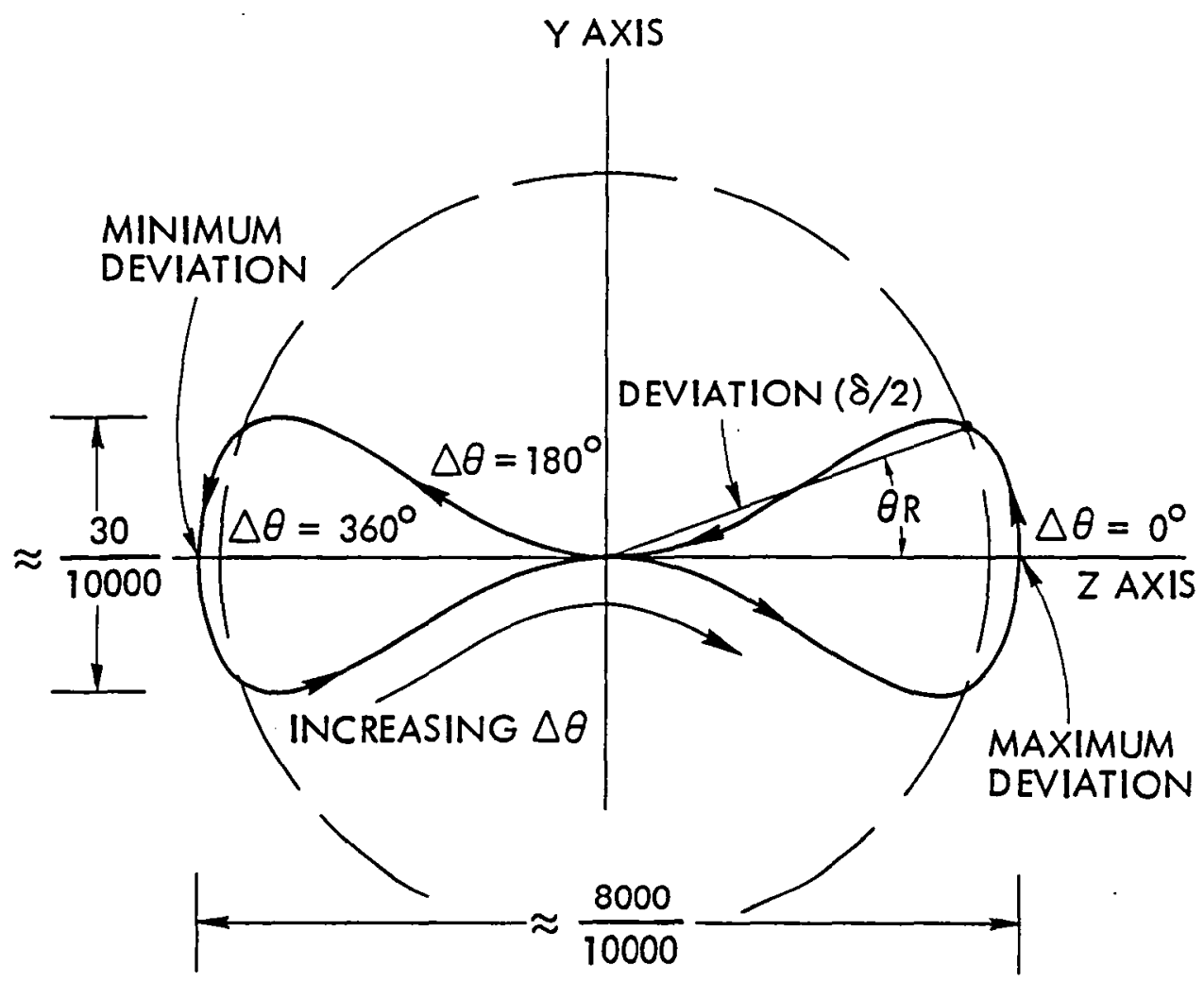

Figure 4. A closer look at counterrotating equal wedges shows errors of 30 meters at 10,000 meters for a $\operatorname{scan} 8,000$ meters wide. 

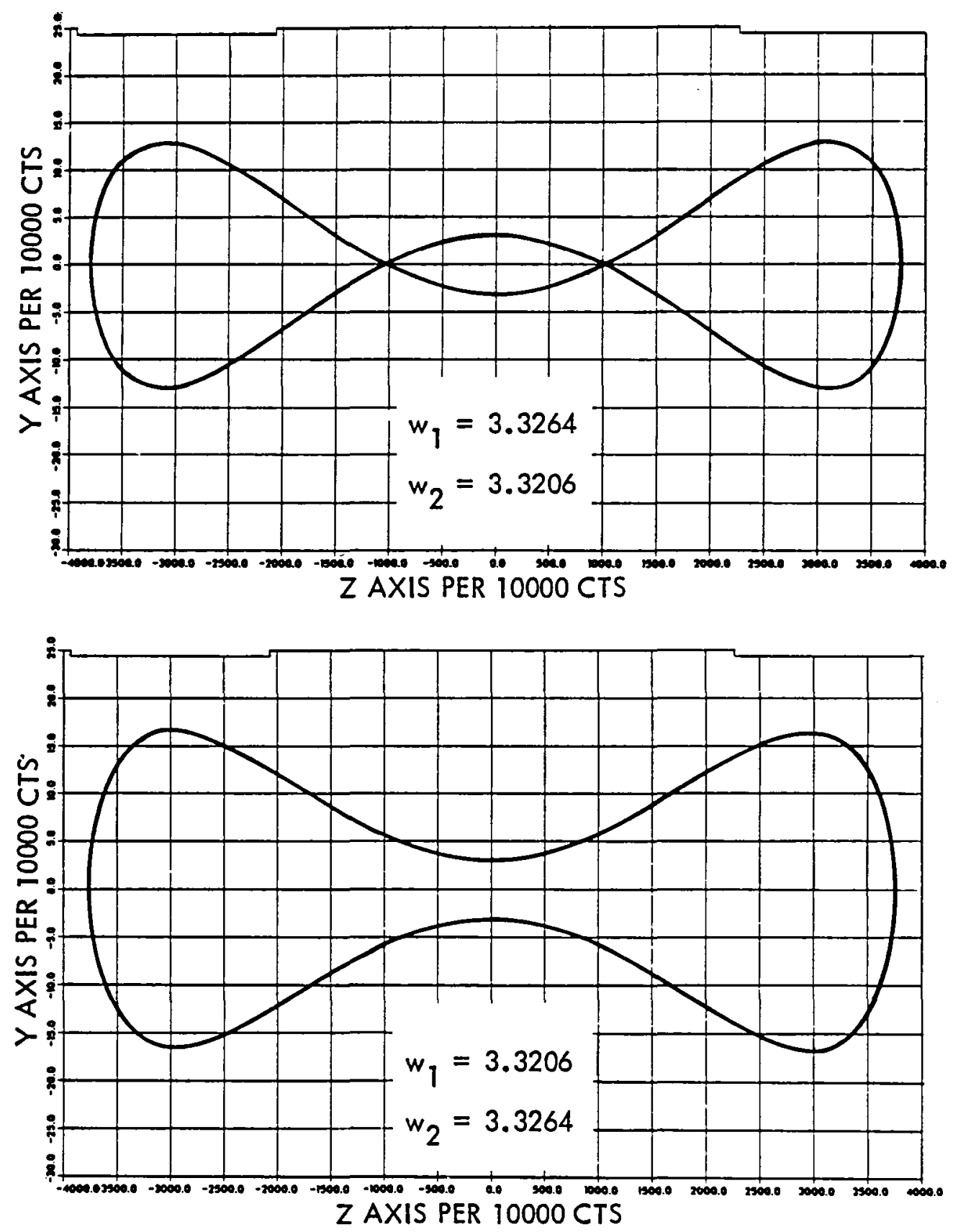

Figure 5. Counterrotating unequal wedges produces greater distortions to the scan generated. 
and its rotation about that axis. The line scan shown earlier may be used to generate any deviation given by

$$
\operatorname{dev}=\cos ^{-1}\left(\frac{x}{\sqrt{x^{2}+y^{2}+z^{2}}}\right)
$$

The key to the algorithm is that the equation for the deviation as a function of $\theta_{2}-\theta_{1}$ can be inverted. If one of the wedge angles is known, this inversion also provides a rotation angle. The second step is to rotate both wedges by the additional amount required to achieve the desired rotation.

The two steps are defined precisely as follows. It is convenient to assume that $\theta_{1}=0^{\circ}$ initially. Then the beam $\hat{s}_{1}=$ $1 \hat{i}+0 \hat{j}+0 \hat{k}$ is refracted through $W_{1}$, whose unit normal is given by $\hat{N}_{1}=\left(\cos w_{1}\right) \hat{i}+\left(\sin w_{1}\right) \hat{j}$. The vector equation for $a$ refracted peam is given by equation (1). Substituting $\hat{S}_{1} \cdot \hat{N}_{1}=$ $\cos w_{1}$, the beam refracted through the first wedge is described by:

$$
\begin{aligned}
\hat{s}_{2}= & r_{1} \hat{i}+\left\{\sqrt{1-r_{1}^{2}\left(\sin ^{2} w_{1}\right)}-r_{1} \cos w_{1}\right\} \\
& {\left[\left(\cos w_{1}\right) \hat{i}+\left(\sin w_{1}\right) \hat{j}\right] . }
\end{aligned}
$$

As expected, there is no $z$ component, since $\theta_{1}=0^{\circ}$.

To derive an equation for $\hat{\mathrm{S}}_{3}$, the output vector, and solve that equation for the rotation angles of the wedges, equation (4) 
is used again in equation (1), along with $\hat{N}_{2}$, calculated using $\theta_{2}=\Delta \theta$, the difference angle. This, in effect, is the same as counterrotating the two wedges except that the frame of reference rotates with $\theta_{1}$ (see Figure 6 ). $\hat{N}_{2}$ is given as

$$
\hat{N}_{2}=\left(\cos w_{2}\right) \hat{i}+\left(\sin w_{2} \cos \Delta \theta\right) \hat{j}+\left(\sin w_{2} \sin \Delta \theta\right) \hat{k} \text {. }
$$

Since, by definition, the final unit vector along the beam from the second wedge will have an $x$ component $x_{3}=\cos ($ dev $)$,

$$
\begin{aligned}
\cos (\mathrm{dev})= & r_{2} x_{2} \\
& +\left\{\sqrt{1-r_{2}^{2}\left[1-r_{2}^{2}\right]\left[1-\left(\hat{S}_{2} \cdot \hat{N}_{2}\right)^{2}\right]}-r_{2} \hat{S}_{2} \cdot \hat{N}_{2}\right\} \cos w_{2},
\end{aligned}
$$

which is related to the angle $\Delta \theta$ only by its dependence on $\hat{S}_{2} \cdot \hat{N}_{2}$. This dot product is obtained using the two-step process shown below. Defining the quantity in brackets in equation (6) as

$$
\begin{aligned}
\delta & =\frac{\cos (\mathrm{dev})-r_{2} x_{2}}{\cos w_{2}} \\
& =\frac{\cos (\mathrm{dev})-r_{2}\left[r_{1} \sin ^{2}\left(w_{1}\right)+\cos \left(w_{1}\right) \sqrt{1-r_{1}^{2} \sin ^{2} w_{1}}\right]}{\cos w_{2}},
\end{aligned}
$$

and solving equation (6) for $\hat{S}_{2} \cdot \hat{N}_{2}$, 


$$
\begin{aligned}
& \theta_{1} \equiv 90^{\circ} \\
& \theta_{2}=90^{\circ}-\Delta \theta \\
& \quad 0^{\circ} \leq \Delta \theta \leq 360^{\circ}
\end{aligned}
$$

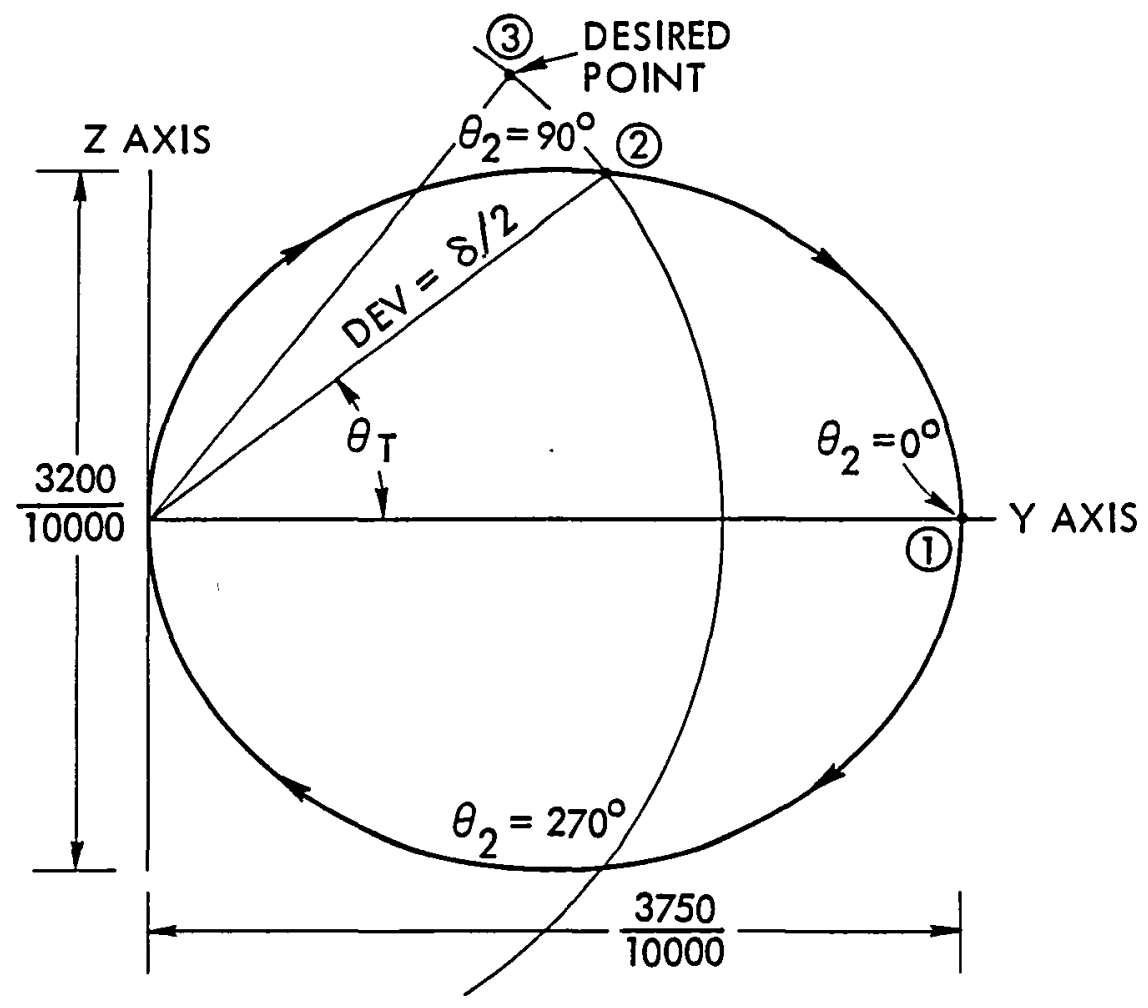

Figure 6. Rotating the second wedge independentiy produces the ellipse shown. The desired point can then be arrived at by rotating the wedges simultaneously. 


$$
\hat{s}_{2} \cdot \hat{N}_{2}=\frac{1-r_{2}^{2}-\delta^{1}}{2 \delta r_{2}}
$$

Recalling equation (5) for $\hat{N}_{2}$, the difference angle is given by

$$
\Delta \theta=\cos ^{-1}\left\{\frac{\left[\hat{S}_{2} \cdot \hat{N}_{2}-x_{2} \cos w_{2}\right]}{y_{2} \sin w_{2}}\right\}
$$

Since the initial condition was $\theta_{1}=0^{\circ}$, the beam has been rotated by an amount equal to the angle between the final $z$ and $y$ components, $\theta_{T}=\tan ^{-1}\left(z_{3} / y_{3}\right)$; the desired rotation, $\theta$, may be achieved by setting $\theta_{1}=\theta-\theta_{T}$ and $\theta_{2}=\theta_{1}+\Delta \theta$.

\section{ALIGNMENT AND CALIBRATION}

The initial application of this system was to position a doppler lidar beam in order to measure wind fields from an aircraft. ${ }^{4}$ On an aircraft, there are several reference frames which may be useful. Transforming from one frame to another involves only the rotation about the three primary axes of the aircraft itself. These rotations, shown in Figure 7, are defined individually. The heading is a negative rotation (using the right-hand rule) about the $y$ axis; heading is defined by the convenient compass angles referenced to true north. The pitch is a negative rotation about the $x$ axis; a positive pitch indicates an upward movement of the nose of the plane. The roll is a positive rotation 


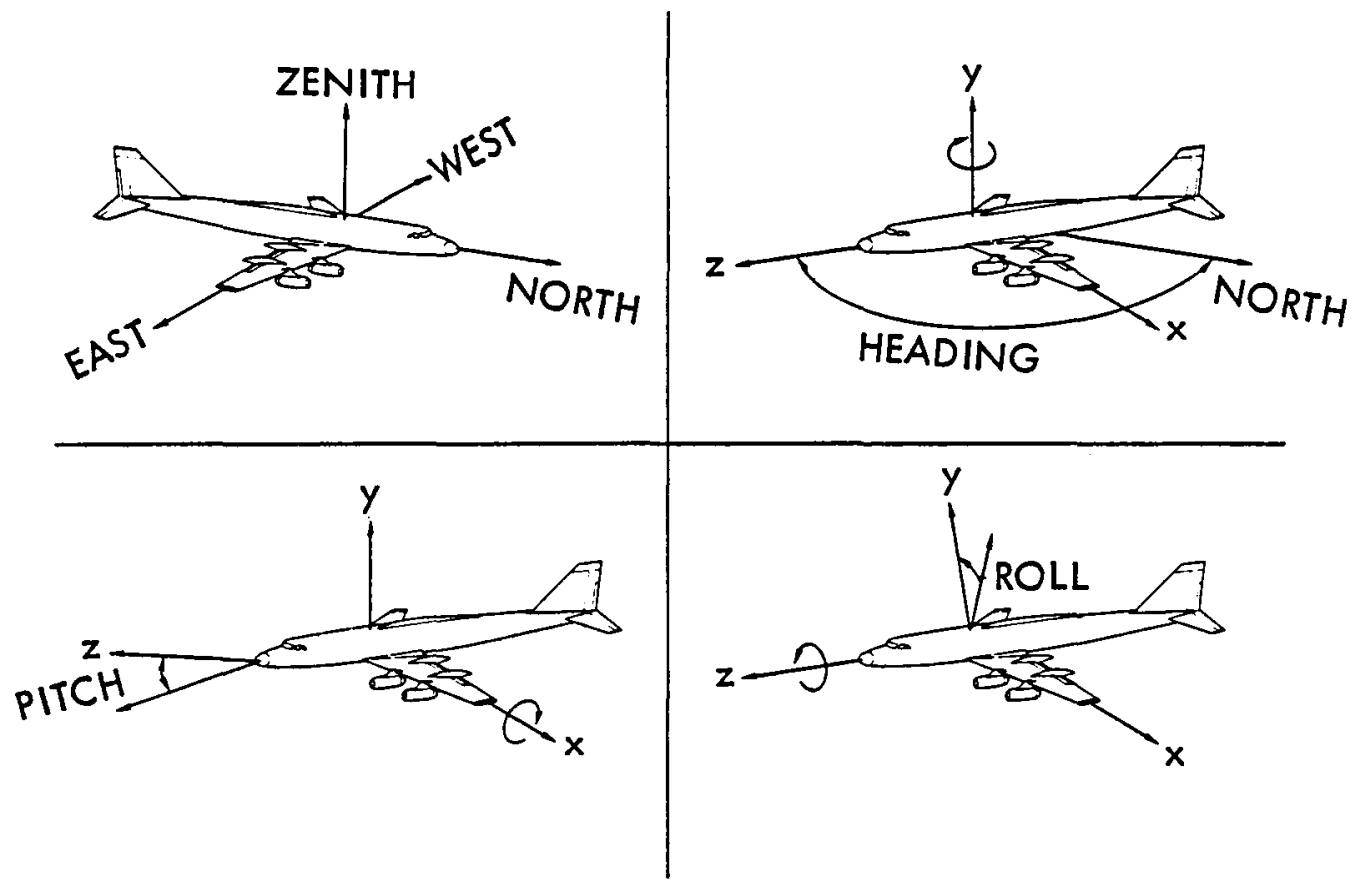

Figure 7. Calculating aircraft alignment involves three rotations about the coordinate systems of heading, pitch and roll. 
about the $z$ axis; a positive roll indicates that the right wing of the aircraft has been moved down. Scanner alignment is done in reference to the aircraft, while points are surveyed in the earth reference system. In order to perform the necessary calculation for alignment, it is necessary to convert the measurement points from earth to aircraft coordinates. Then, just previous to comparison, they must be converted back into earth coordinates. The earth-to-aircraft process involves two steps: first, they must be "unheaded"-rotated positively about the $y$ axis, an amount equal to $90^{\circ}$ less the measured true heading angle; second, the vectors must be "unrolled"-rotated negatively about the $z$ axis, an amount equal to the measured roll angle. It should be clear to see that reversing these calculations affects the vectors to return to earth coordinates. The apparent omission of pitch correction is deliberate; the rotation of the wedges accounts for this movement.

There are at least six critical parameters for the correct alignment of the scanner: $w_{1}, w_{2}, \theta_{1}, \theta_{2}$, ROLL and THG (true heading). In order to precisely position an output beam, it is necessary to align the axis of the scanner and the $0^{\circ}$ reference marks of both wedges with the three axes of the inertial navigation system, as well as to ascertain the wedge angles of both wedges. Since it would be difficult to locate the required reference surfaces and perform the installation accurately, a 
method has been developed to determine these angles from measurements made after the scanner is installed in the aircraft; specifically, a program has been written which calculates the wedge and rotation angles as well as the errors in roll and heading using four selected scan vectors at which the beam is positioned.

It would be possible to use the four measured parameters, $\theta_{\text {ind }}$, ROLL ${ }_{\text {ind }}$, PITCH ${ }_{i n d}$, and THG $_{\text {ind }}$ (where the subscript "ind" denctes "indicated"), with the measured vectors $\hat{s}_{1}$ through $\hat{s}_{4}$ to calculate errors between actual and predicted lines of sight, and through iteration directly adjust the six parameters. This process has two primary faults: the mathematics are complex, and in general, all the vectors are affected by a change in any of the six parameters. To alleviate these problems, a set of six linear combinations of the parameters was chosen, $P_{1}$ through $P_{6}$, and with them six check parameters, $\mathrm{CK}_{1}$ through $\mathrm{CK}_{6}$, so that each check is most strongly dependent on its own scan parameter. This process, which was accomplished through intuition and trial and error, is similar to the diagonalization of a matrix representing a system of linear equations. This corresponding dependence makes it possible to adjust the scan parameters, calculate new vectors $S_{1}$ through $S_{6}$ and then their trial check parameters, and finally compare these check parameters to those of the actual vectors. In this way, each scan parameter is adjusted to reduce its own check parameters with a minimum of interaction among the parameters. 
The two sets are listed below.

\section{Scan Parameter}

$P(1)=\left|w_{2}\right|+\left|w_{1}\right|$

Total wedge angle
Check Parameter

$\operatorname{Ck}(1)=\cos ^{-1}\left(\hat{S}_{1} \cdot \hat{S}_{2}\right)$ [signed]

Distance between two points near maximum deviation
$P(2)=\left|w_{2}\right|-\left|w_{1}\right|$

Difference between the two wedge angles
$\operatorname{Ck}(2)=\cos ^{-1}\left(\hat{S}_{3} \cdot \hat{S}_{4}\right)$ [signed]

Distance between two points near minimum deviation

$$
\begin{aligned}
C k(3)= & \frac{D_{1}+D_{2}}{2} \text { where } D_{1}=\cos ^{-1} \\
& \left(\hat{S}_{1} \cdot \hat{S}_{3}\right)-\cos ^{-1}\left(\hat{S}_{3} \cdot \hat{S}_{2}\right) \text { and } \\
& D_{2}=\cos ^{-1}\left(\hat{S}_{1} \cdot \hat{S}_{4}\right)-\cos ^{-1} \\
& \left(\hat{S}_{4} \cdot \hat{S}_{2}\right)
\end{aligned}
$$

Difference in deviation between two extremes of the scan

$P(4)=\theta_{2}+\theta_{1}$

$\begin{aligned} C k(4)= & \tan ^{-1}\left(y_{1}-y_{2}\right) / \text { DIST where } \\ & \text { DIST }=\sqrt{\left(x_{1}-x_{2}\right)^{2}+\left(z_{1}-z_{2}\right)^{2}}\end{aligned}$

Height difference between horizontal extremes of the scan
$C k(5)=\left[\cos ^{-1}\left(y_{3}\right)+\cos ^{-1}\left(y_{4}\right)\right] / 2$ Mean elevation angle near center of scan

Roll error Indicated roll actual heading

$P(6)=$ Actual heading $-C k(6)=\left[\cos ^{-1}\left(x_{3}\right)+\cos ^{-1}\left(x_{4}\right)\right] / 2$ Indicated heading

Heading error

Mean heading near center of scan 
These six check parameters are calculated from the four surveyed lines of sight, and those values are compared to the check parameters of scan vectors calculated from the scan parameter values. The errors between the two sets of checks are reduced by correcting the scan parameters, one at a time, each using its own check, until by successive iteration a final set of scan parameters is reached. This process has been simulated at Raytheon Company's Scientific Computer Center, and the results are shown in Figure 8.

It was assumed above that the incident beam was parallel to the axis of rotation. The case for which this is not true has also been investigated, and it was discovered that most of the errors created by an initial beam rotation around the $y$ and $z$ axes are absorbed later in the scan parameter values of heading and roll errors, respectively. In other words, the deviation produced by the wedges is almost independent of the angle of incidence for the small alignment errors investigated. The following values were calculated to support these claims.

$\begin{array}{lll}\text { No Rotation Rotation } 1^{\circ} & \text { Rotation } 1^{\circ} \\ \text { Correct P's } & \begin{array}{l}\text { Robout } y \text { axis } \\ \text { about } z \text { axis }\end{array}\end{array}$

Wedge Sum

Wedge Difference Rotation Difference Rotation Sum Roll Error Heading Error
6.725

$-0.04$

1.6

0.6

0.33

0.75
6.730

$-0.035$

1.8

0.7

0.335

1.79
6.725

$-0.04$

1.6

0.6

1.37 


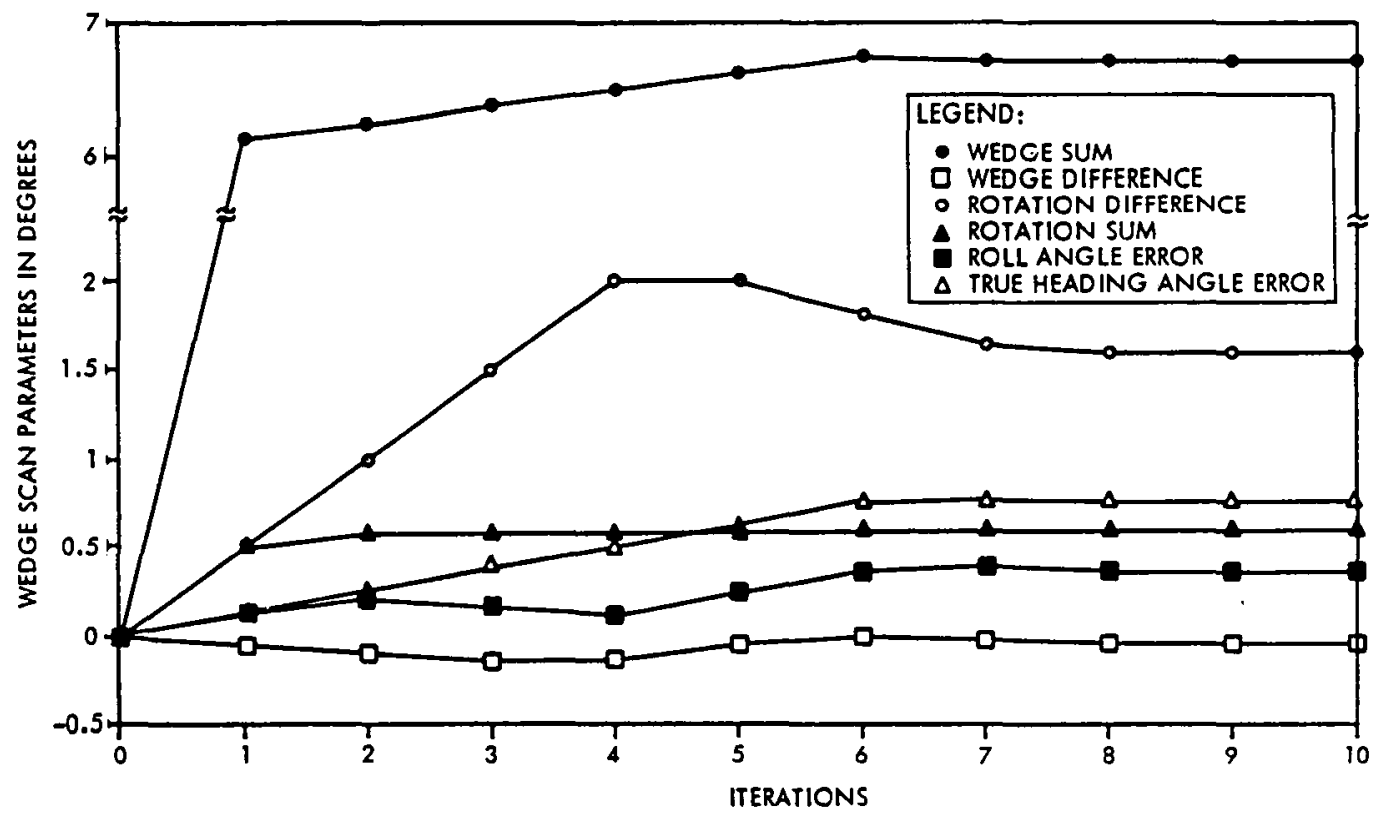

Figure 8. As the iterations progress, each of the parameters approaches its correct value. 
As shown, the rotation about the $y$ axis causes a corresponding increase in the heading error, with slight errors also occurring in the other parameters. These additional errors are expected, since the points selected for the calibration are all close to the $x-z$ plane. The roll error absorbs completely the error from the rotation about the $z$ axis.

Using these two misaligned parameter sets, scatter plots of errors (see Figures 9 and 10) showed that position errors at 10,000 meters of range were less than 16 meters. The vertical axis on the plots corresponds to the $z$ axis. The circles show the outer limits of the $20^{\circ}$ scan; therefore, the points inside the circles represent all points reachable by the scanner. Each point in a circle is represented by a box containing 0 to 161 ines. The number of 1 ines is proportional to the error in positioning the scanner at that point. A black box, which contains all 16 lines, represents the maximum error in meters at 10,000 meters for each plot, and is shown in the legend.

\section{1981 TEST RESULTS}

In 1981, a dual-wedge lidar system was developed for NASA's Severe Storms Doppler Lidar Flight Program. This system used two germanium wedges to point a $\mathrm{CO}_{2}$ laser beam out the left side of a CV-990 aircraft in order to collect wind field data. This was done by collecting line-of-sight doppler velocity data with two sequential sets of pulse trains along lines of sight $20^{\circ}$ 


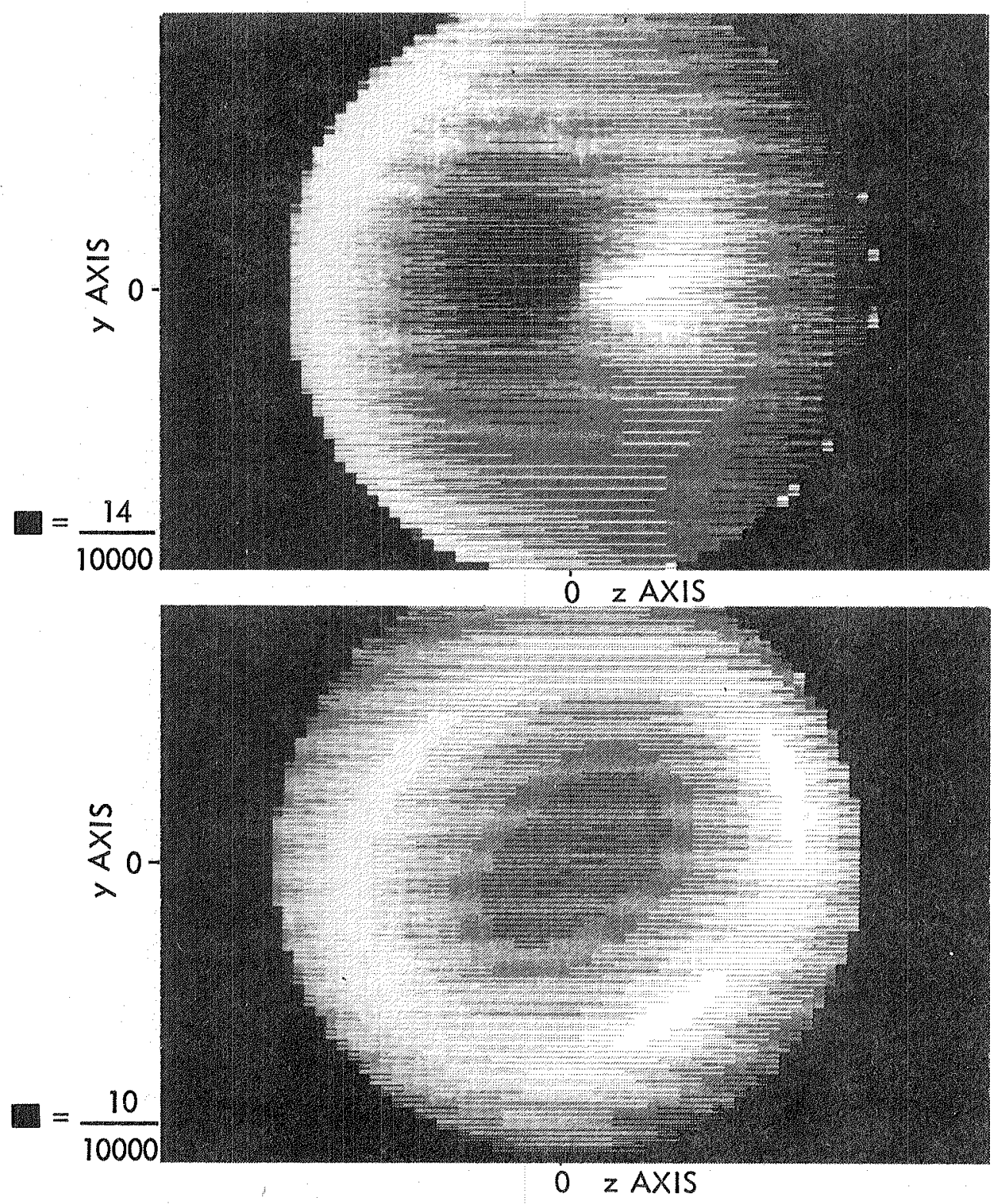

Figure 9. Results better than 14 and 10 meters at 10,000 meters are acquired with the two misaligned parameter sets if the rotation angles are unrounded. 


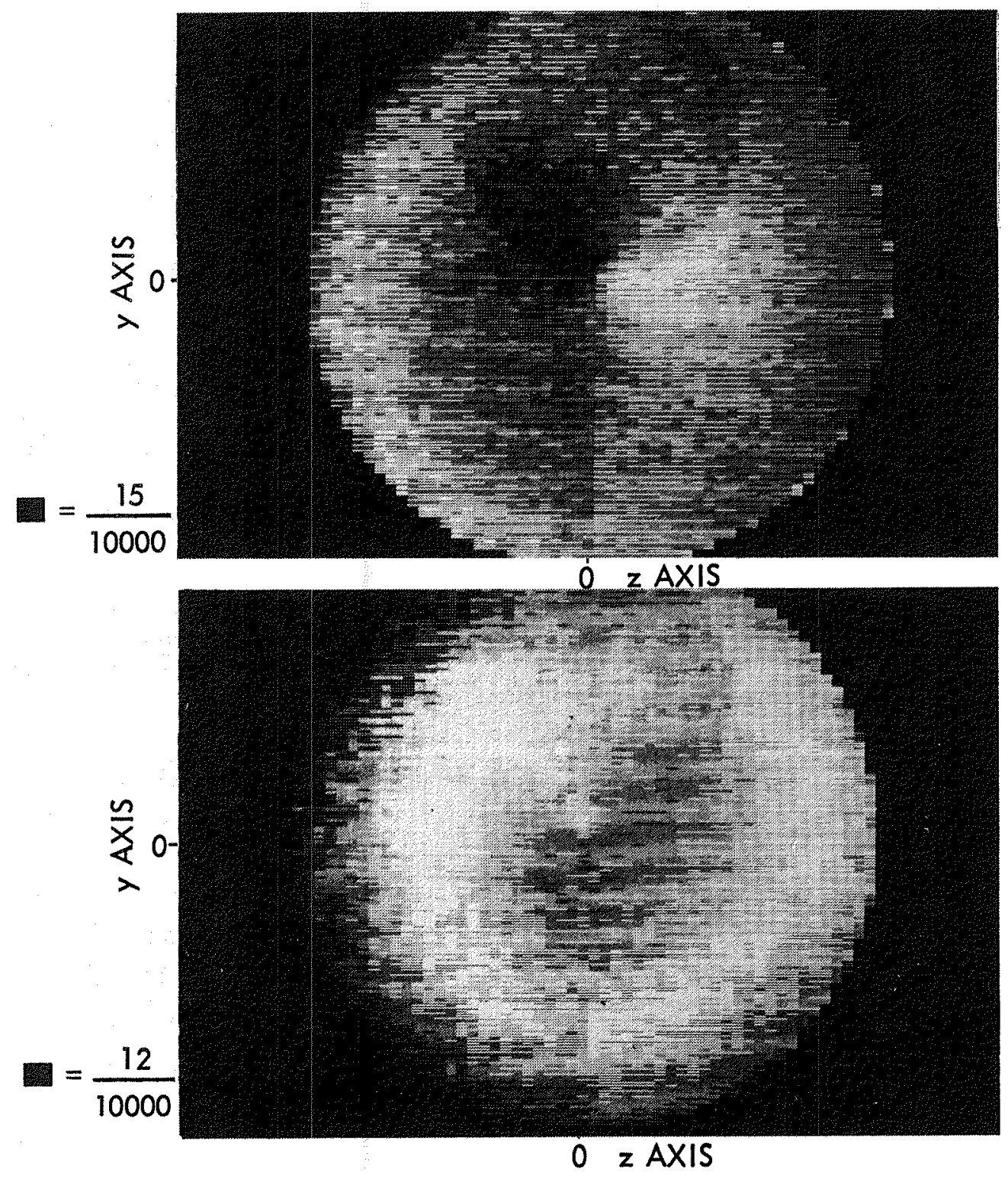

Figure 10. Due to the one-tenth degree quantization in positioning the scanner, the actual errors are 15 and 12 meters at 10,000 meters. 
forward and $20^{\circ}$ aft of the nominal left-looking line every 1.2 seconds. Then a grid of wind velocity vectors was created by combining the doppler measurements along the fore and aft lines at all their intersecting points (see Figure 11).

Previous to the system's installation, the two wedge angles were measured to be $w_{1}=3.3264^{\circ}$ and $w_{2}=3.3206^{\circ}$. Upon installation, the system was tested for alignment by directing the beam to surveyed points and collecting the associated parameter values (see Figure 12). From this set of points, four were chosen to be processed through the alignment program to determine the correct parameters. Because the proximity of the two centermost points prevented the program from successfully finding the wedge-difference parameter $P_{2}$, this parameter was set to zero throughout the run, which is equivalent to assuming equal wedge angles. The alignment program arrived at values of $w_{1}=w_{2}=$ $3.3275^{\circ}$, which are $0.21 \%$ and $0.03 \%$ from the values measured in the laboratory.

As noted before, the two innermost vectors were too close to each other for best possible results. For the greatest accuracy, the two outermost points should be separated by no less than $4.6^{\circ}$ and the two innermost points by at least $0.35^{\circ}$. Even these nonideal choices have been demonstrated to produce successful results in the model tests. 


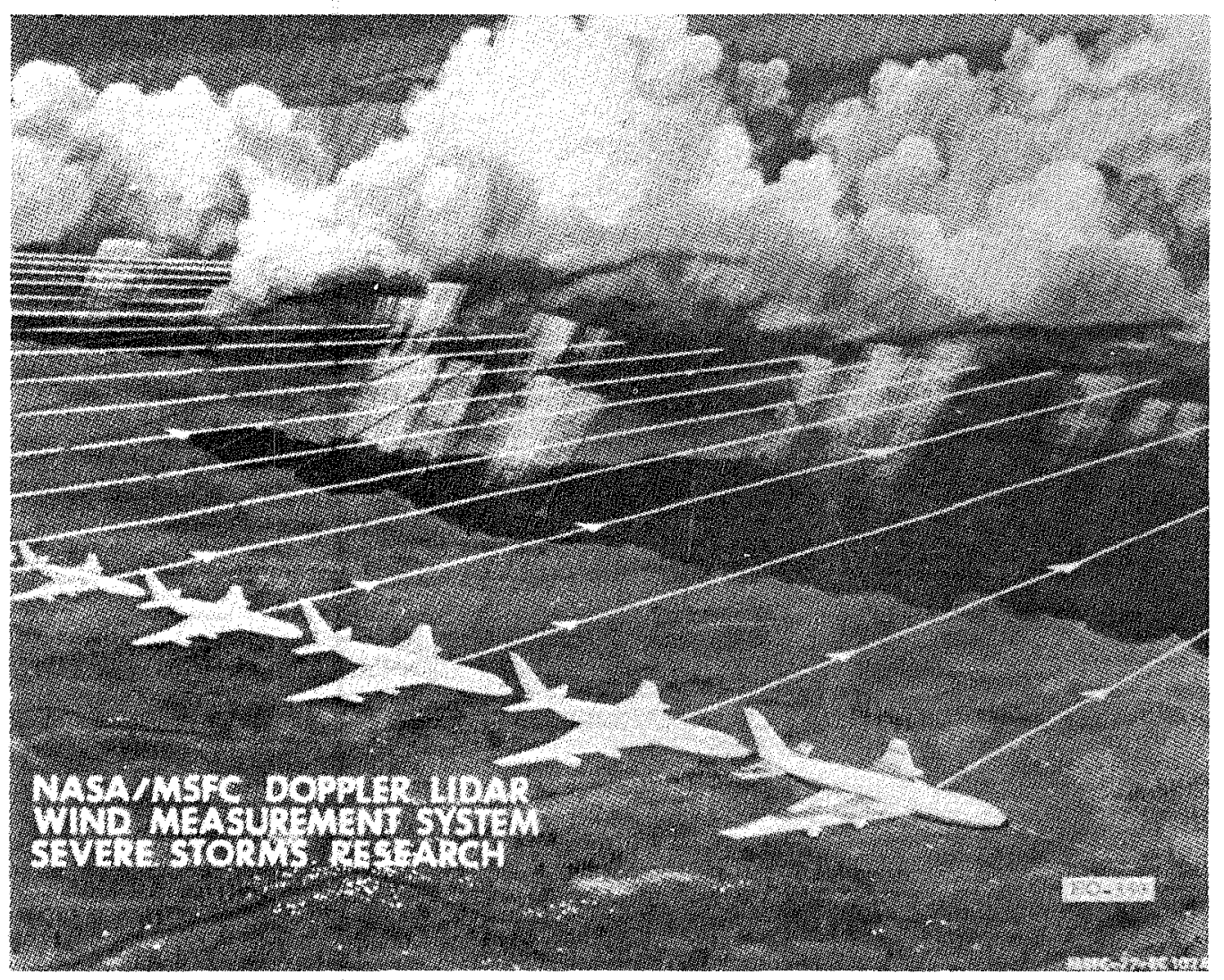

Figure 11. Fore and aft looking scans create a grid of wind velocity returns in the Severe Storms measurement system. 


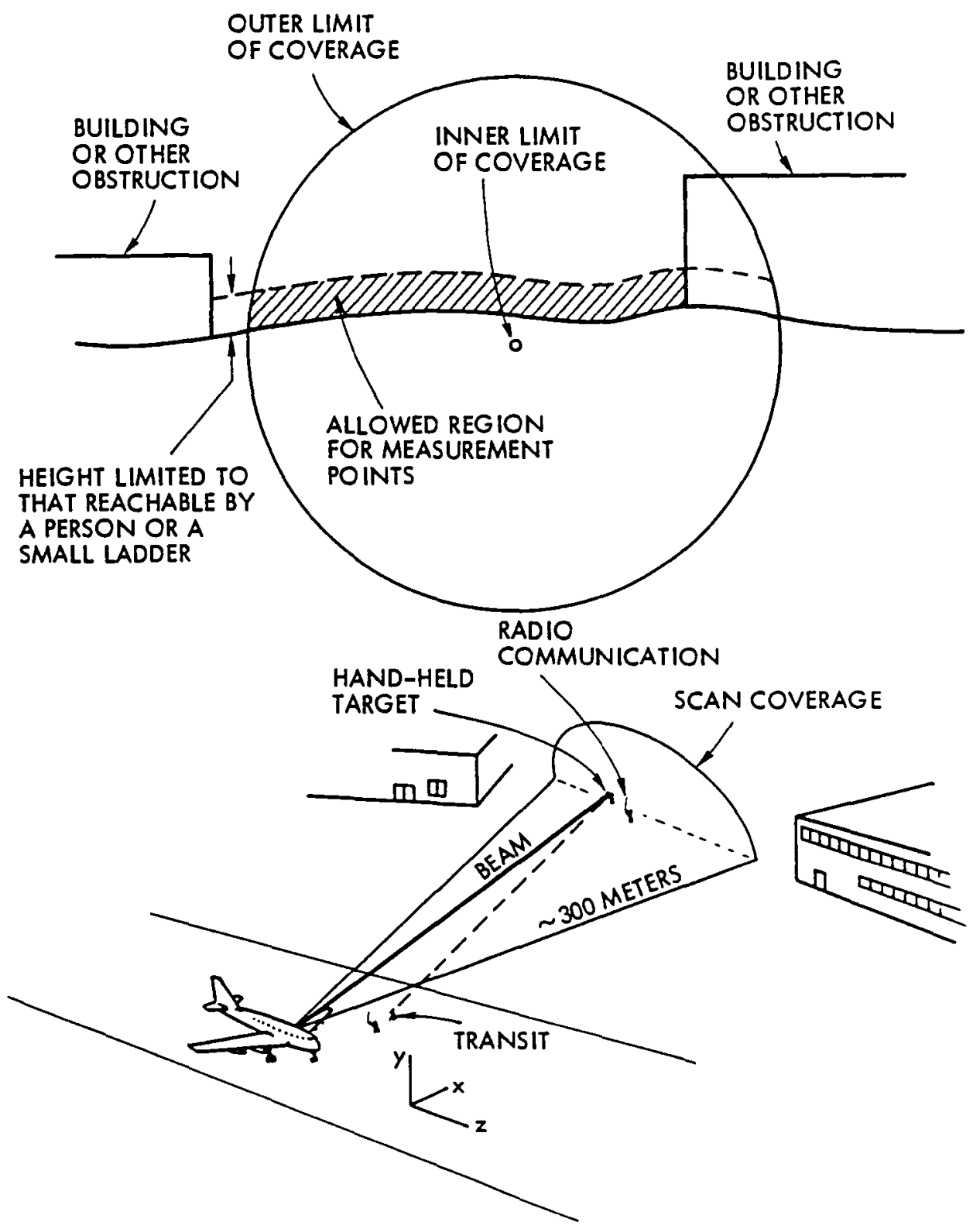

Figure 12. The acquisition and selection of data points is restricted by scanner coverage, physical obstructions, and human limitations. 


\section{CONCLUSION}

The Severe Storms tests, which ran from 12 June 1981 to 30 July 1981 , used the dual-wedge system successfully to position a laser beam for vector wind velocity measurements. An error in the data that was originally attributed to misalignment seems to have been caused at least in part by the straight line deviations outlined in Section 2. Overall, the system performed admirably, and the same dual-wedge system will be on board for the next flights beginning in the summer of 1984 . The equations developed here for positioning and alignment will be used for these tests and will be evaluated in a ground-based experiment in early 1984 . 


\section{REFERENCES}

1. C. DiMarzio, C. Harris, J.W. Bilbro, E.A. Weaver, D.C. Burnham, and J.N. Hallock, "Pulsed Laser Doppler Measurements of Wind Shear," Bull. Am. Meteor. Soc., 60, No. 9, 1061-1066 (September 1979).

2. C. DiMarzio, M. Krause, R. Chandler, J. O'Reilly, K. Shaw, J. Bilbro, E. Weaver, "Airborne Lidar Dual Wedge Scanner," presented at Eleventh International Laser Radar Conference, University of Wisconsin-Madison, P. 123, NASA CP-2228, June 21-25, 1982.

3. M. Born and E. Wolf, Principles of Optics, 2nd ed. (MacMillan, New York, 1964), p. 125.

4. Charles A. DiMarzio and James W. Bilbro, "An Airborne Doppler Lidar," presented at Heterodyne Systems and Technology Conference Part II, Williamsburg, Virginia, p. 529, NASA CP-2138, Part 2, March 25-27, 1980. 


\begin{tabular}{|c|c|}
\hline 2. GOVERNME NT ACCESSION NO. & 3. RECIPIENT'S CATALOG NO. \\
\hline \multirow{2}{*}{$\begin{array}{l}\text { 4. TITLE AND SUBtitLe } \\
\text { Precision Pointing Using a Dual-Wedge Scanner }\end{array}$} & $\begin{array}{l}\text { 5. REPORT DATE } \\
\text { NOV Ember } 1985\end{array}$ \\
\hline & 6. PERFORMING ORGANIZATION CODE \\
\hline $\begin{array}{l}\text { 7. AUTHOR(S) } \\
\text { Christopher T. Amirault and Charles A. DiMarzio }\end{array}$ & 8.PERFORMING ORGANIZATION REPORT \# \\
\hline \multirow{3}{*}{$\begin{array}{l}\text { 9. PERFORMING ORGANIZATION NAME ANO ADDRESS } \\
\text { Raytheon Company } \\
\text { Equipment Development Laboratories } \\
\text { Electro-Optics Systems Laboratory } \\
\text { Sudbury, MA } 01776\end{array}$} & $\begin{array}{l}\text { 10. WORK UNIT. NO. } \\
\text { M-478 }\end{array}$ \\
\hline & \multirow{4}{*}{$\begin{array}{l}\text { 11. CONTRACT OR GRANT NO. } \\
\text { NAS8-33120 } \\
\text { 13. TYPE OF REPORT \& PERIOD COVERED } \\
\text { Contractor Report }\end{array}$} \\
\hline & \\
\hline \multirow{3}{*}{$\begin{array}{l}\text { 12. SPONSORING AGENCY NAME AND ADDRESS } \\
\text { Nationa1 Aeronautics and Space Administration } \\
\text { Washington, D. C. } 20546\end{array}$} & \\
\hline & \\
\hline & 14. SPONSORING AGENCY CODE \\
\hline
\end{tabular}

15. SUPPLEMENTARY NOTES

Technical Monitor: James W. Bilbro, George C. Marshall Space Flight Center, AL. This is a topical report.

16. ABSTRACT

A system has been developed for calibrating and precisely pointing a germanium dual-wedge scanner for a $\mathrm{CO}_{2}$ doppler lidar from an airborne platform. This paper describes the equations implemented in pointing the scanner as well as those in the iterative calibration program, which combines available data with estimated parameters of the scanner orientation relative to the axes of the aircraft's inertial navigation system to arrive at corrected scanner parameters. In addition, the effect of specific error conditions on program performance and the results of the program when used on 1981 test data are investigated.

\section{KEY WORDS}

Dual-wedge scanner Iterative calibration program $\mathrm{CO}_{2}$ doppler lidar
18. DISTRIBUTION STATEMENT

Unclassified-Unlimited

STAR Category: 43
19. SECURITY CLASSIF. ( $\alpha$ thle ropart)

Unclassified
20. SECURITY CLASSIF. (ol thie page)

Unclassified \begin{tabular}{|l|l|} 
21. NO. OF PAGES & 22. PRICE
\end{tabular}

35

A03 


\section{End of Document}

